\title{
Bacteriological and Mycological profile of Chronic Suppurative Otitis Media among patients visiting Dhulikhel Hospital
}

\author{
Krista Vaidya, ${ }^{1 *}$ Surendra Kumar Madhup, ${ }^{2}$ Bikash Lal Shrestha, ${ }^{2}$ A Gautam, ${ }^{1}$ Nuchhe Ratna \\ Tuladhar $^{2}$
}

BACKGROUND: Chronic suppurative otitis media (CSOM) is an inflammation of the middle ear and mastoid mucosa with perforation of tympanic membrane. Mainly disease of developing countries like Nepal, CSOM results because of illiteracy, poverty and poor hygiene. Haphazard use of antibiotics and increasing use of newer one has led to persistent change in microbial flora. The aim of this study is to determine the incidence of CSOM and its causative agents.

METHODS: The study included 123 samples from 105 patients attending ENT department of Dhulikhel hospital. Samples were processed in microbiology department for both bacteria and fungi using standard operating protocol. Antibiotic susceptibility testing was performed for all bacterial isolates by Kirby Bauer disc diffusion method and the result were interpreted according to clinical and laboratory standard institute (CLSI) guideline.

RESULTS: Out of 105 patients, 55 were male and 50 female patients. Highest incidence of CSOM was observed between 1-10 years of age group. Of the total 123 samples taken from 105 patients, 106 showed microbial growth. Gram positive bacteria predominated and the most common bacteria isolated were $S$. aureus $54.55 \%$ followed by Proteus spp. $13.64 \%$ and $P$. aeruginosa $12.73 \%$. Among the fungi, the most predominant was $A$. fumigatus $39 \%$ followed by $A$. niger 29\%, $C$. albicans $26 \%$ and $A$. flavus $6 \%$. Gentamycin was the most susceptible antibiotic. $S$. aureus were sensitive to Cloxacillin and Gentamycin, whereas Proteus spp. was most sensitive to Ceftriaxone and Norfloxacin. P. aeruginosa was $100 \%$ sensitive to Amikacin.

CONCLUSION: $S$. aureus was the most predominant organism followed by Proteus spp. and the drug of choice was Gentamycin.

Key words: Chronic suppurative otitis media, bacteria, fungi, antibiotic

(C) 2015 Nepalese Association for Clinical Chemistry

\section{Introduction}

Chronic suppurative otitis media (CSOM) is characterized by a persistant discharge from the middle ear through a tympanic membrane perforation [1].The disease is classified into tubotympanic and atticoantral depending upon where the disease affects [2].

It is an important cause of preventable hearing loss, particularly in the developing world [1]. Annually it affects between 65 to 330 million individuals and cause 28000 deaths per annum [1]. In Nepalese context prevalence of CSOM is $5 \%$ which is higher than other countries and more than $55 \%$ of these cases occur in school going children, most of them belonging to the lower socio-economic class [3].

Most commonly isolated aerobic bacteria in CSOM are Pseudomonas aeruginosa, Escherichia coli, Staphylococcus aureus, Streptococcus pyogenes, Proteus mirabilis, Klebsiella spp [4]. Fungal infection of the middle ear and external auditory meatus are common as fungi thrive well in moist pus and the mostly isolated fungi are Candida species and Aspergillus species. But the type of organism isolated varies between the geographical areas and other factors [5].

It is nowadays rare for an otologist to see ears with discharge that have not already had the bacterial flora modified by antibiotic therapy since most patients attend the hospital very late when treatment becomes a problem and cultures are frequently sterile. This may be because of microbial resistance to these antibiotics thereby suggesting their failure leading to continuation of purulent discharge in the discharging ear [6]. Hence, providing pattern of modern day isolates and their sensitivity toward antibiotics was the centre of this study.

\section{Methods}

Cross sectional descriptive analytical study was carried out at the department of microbiology in Dhulikhel hospital, Kathmandu University Hospital from April 2011 to March 2012. One 
hundred and five clinically diagnosed CSOM patients, having ear discharge for more than 3 months with unilateral or bilateral disease attending out-patient department of ENT, were included in this study. An otolaryngologist diagnosed and evaluated CSOM by otoscopic examination. Then using sterile cotton swab, ear discharge was collected through perforation in the tympanic membrane via sterile aural speculum to avoid contamination from the skin of the auditory canal. In case of bilateral infection, samples were taken from both ears. So, in total 123 middle ear samples were collected from the total 105 CSOM patients. Samples were then immediately transported to Microbiology department.

Each patient requested for culture was directly interviewed for his or her clinical history during sample collection. The preformed performa was filled documenting age, sex, and clinical information including side of ear affected, and types of CSOM.

Samples were inoculated into sheep blood agar (SBA), MacConkey agar (MA) and Chocolate agar (CA) for isolation of aerobic bacteria and incubated aerobically at $37^{\circ} \mathrm{C}$ for 24 hours. For isolation of fungi, samples were inoculated into two sabouraud dextrose agar (SDA); one incubated at $25^{\circ} \mathrm{C}$ and other incubated at $37^{\circ} \mathrm{C}$ for upto 7 days. Antibiotic susceptibility testing of bacterial isolates was performed on muller hinton agar (MHA) by Kirby Bauer disc diffusion method using CLSI guideline [7]. Results obtained were analysed by SPSS version 16 .

\section{Results}

Out of 105 cases studied, 78(74\%) had tubotympanic type of CSOM and $27(26 \%)$ had atticoantral type. Unilateral infection was seen in $87(82.85 \%)$ patients and bilateral in 18 (17.15\%). Of the cases studied, 55 (52.38\%) were male and $50(47.61 \%)$ female. Similarly, higher incidence of CSOM was seen in 1-10yrs of age group and it decreases as the age increases. Figure 1 shows the age and gender wise distribution of CSOM. Among the 105 patients, no growth was seen in 14 patients. Of the 91 patients showing microbial growth, 46 were male and 45 female. Age-wise high microbial growth was observed in 11-20 years (24) and 1-10 years (23) followed by 21-30 years (16) and age group
$31-40$ and $>40$ showed equal number of growth i.e. 14 .

\section{Fig 1. Age and gender wise distribution of CSOM}

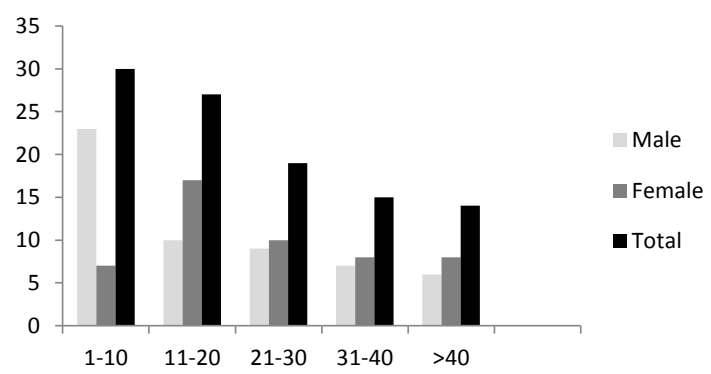

Of the 123 samples collected from 105 patients, 106 showed microbial growth and 17 showed no growth. Of the total growth, 64 were pure bacterial isolates, 9 pure fungal and 33 mixed types. Total 141 microorganisms were isolated in this study, gram positive bacteria predominated; Staphylococcus aureus being the most predominated one followed by Proteus spp and Pseudomonas aeruginosa respectively. In case of fungal isolates, Aspergillus species predominated of which A. fumigatus prevailed (Table 2).

Out of 63-gram positive bacteria isolated, 59 (93.65\%) were susceptible toward cloxacillin, 58 $(92.06 \%)$ toward gemtamycin and $56(88.89 \%)$ toward chloramphenicol. 59 (93.65\%) grampositive bacteria showed resistance against Penicillin.

Antibiotic susceptibility testing done for gramnegative bacteria except Pseudomonas aeruginosa showed higher susceptibility toward gentamycin $(91.9 \%)$, ceftriaxone $(87.9 \%)$, norfloxacin $(84.85 \%)$ and ciprofloxacin $(81.8 \%)$.

Staphylococcus aureus the most predominant organisms isolated in this study was found to be most sensitive toward cloxacillin and gentamycin whereas most of the isolates were resistant to penicillin. Out of 60, Staphylococcus aureus isolated, $4(6.67 \%)$ were found to be methicillin resistant (Table 3). Similarly, Proteus species were $100 \%$ sensitive to ceftriaxone and norfloxacin (Table 3). The antibiotic susceptibility testing for Pseudomonas aeruginosa showed $100 \%$ sensitivity toward amikacin (Table 5). 
Table 1. Bacteriological profile in CSOM

\begin{tabular}{llcc}
\hline \multirow{2}{*}{ Type of bacteria } & \multicolumn{1}{c}{ Organism } & $\begin{array}{c}\text { No. of isolates } \\
(\mathrm{n}=1 \text { 10) }\end{array}$ & $\%$ \\
\hline \multirow{3}{*}{ Gram positive } & Staphylococcus aureus & 60 & 54.54 \\
& Enterococcus spp. & 1 & 0.91 \\
& Streptococcus pneumoniae & 2 & 1.82 \\
\hline \multirow{3}{*}{ Proteus mirabilis } & 6 & 5.45 \\
& Proteus vulgaris & 9 & 8.18 \\
& Pseudomonas aeruginosae & 14 & 3.64 \\
& Klebsiella oxytoca & 4 & 1.82 \\
& Klebsiella pneumoniae & 2 & 4.54 \\
& Escherichia coli & 5 & 1.82 \\
& Acinetobacter spp. & 2 & 2.73 \\
& Enterobacterspp & 3 & 1.82 \\
\hline
\end{tabular}

Table 2. Fungal profile in CSOM

\begin{tabular}{lcc}
\hline Organism & No. of isolates $(\mathbf{n}=31)$ & $\%$ \\
\hline A. fumigatus & 12 & 38.71 \\
A. niger & 9 & 29.03 \\
A. flavus & 2 & 6.45 \\
C. albicans & 8 & 25.81 \\
\hline
\end{tabular}

Table 3. Antibiotic susceptibility pattern of Staphyloccous aureus

\begin{tabular}{lccc}
\hline Antibiotics $(\mu \mathrm{g})$ & Sensitive (\%) & Resistant (\%) & Intermediate (\%) \\
\hline Cloxacillin $(10)$ & 93.33 & 6.67 & 0 \\
Penicillin (10) & 1.67 & 98.33 & 0 \\
Cefazolin (30) & 73.33 & 16.67 & 10 \\
Erythromycin (15) & 50 & 30 & 20 \\
Gentamycin (10) & 91.67 & 8.33 & 0 \\
Ciprofloxacin (5) & 51.67 & 33.33 & 15 \\
Chloramphenicol (30) & 88.33 & 3.33 & 8.33 \\
\hline
\end{tabular}

Table 4. Antibiotic susceptibility pattern of Proteus spp

\begin{tabular}{lccc|ccc}
\hline \multirow{2}{*}{ Antibiotics $(\mu \mathrm{g})$} & \multicolumn{3}{|c|}{ Proteus mirabilis $(\mathbf{n}=9)$} & \multicolumn{3}{c}{ Proteus vulgaris $(\mathrm{n}-6)$} \\
\cline { 2 - 7 } & $\begin{array}{c}\text { Sensitive } \\
(\%)\end{array}$ & $\begin{array}{c}\text { Resistant } \\
(\%)\end{array}$ & $\begin{array}{c}\text { Intermediate } \\
(\%)\end{array}$ & Sensitive (\%) & Resistant (\%) & $\begin{array}{c}\text { Intermediate } \\
(\%)\end{array}$ \\
\hline Gentamycin (10) & 77.78 & 22.22 & & 100 & & \\
Ciprofloxacin (5) & 100 & & 11.11 & 66.67 & 33.33 & 16.67 \\
Cephalexin (30) & & 88.89 & & 100 & 50 & \\
Ceftriaxone (30) & 100 & & 100 & & \\
Norfloxacin (10) & 100 & 100 & & 66.67 & 33.33 & \\
Tetracycline (30) & & 11.11 & & 50 & 50 & \\
Amoxycyllin (10) & 88.89 & 11.11 & &
\end{tabular}

Table 5. Antibiotic susceptibility pattern of Pseudomonas aeruginosa

\begin{tabular}{lccc}
\hline Antibiotics $(\boldsymbol{\mu g})$ & Sensitive (\%) & Resistant (\%) & Intermediate (\%) \\
\hline Amikacin (30) & 100 & 0 & 0 \\
Ceftazidime (30) & 85.71 & 14.29 & 0 \\
Carbenicillin (100) & 78.57 & 21.43 & 0 \\
Piperacillin (100) & 28.57 & 71.43 & 0 \\
Ciprofloxacin (5) & 92.86 & 0 & 7.14 \\
Gentamycin (10) & 92.86 & 7.14 & 0 \\
\hline
\end{tabular}




\section{Discussion}

In this study male predominance was higher $(52.38 \%)$ than female. It is in accordance to other studies [8-10] but is in contrast to study done by Loy et al and Mansoor et al [11, 12]. Age group 1-10 years had the higher prevalence of CSOM $28.57 \%$. Study done by Shrestha et. Al. and Jha et. Al. also found the similar result $[13,14]$. High prevalence rate in children may be due to multiple reasons as young children and infants may have low resistance and also because of relatively short Eustachian tube. Due to short Eustachian tube, infected material from the nose, adenoids and sinuses passes more readily along the Eustachian tube to the tympanic cavity, particularly during coughing, sneezing, vomiting, and forced feeding commonly practiced in our environment with the child's nose blocked, while being held head down and half prone [15]. It may be also attributed to the fact that they are more prone to upper respiratory tract infections. Furthermore, cold weather predisposes children to upper respiratory tract infection. Poor hygiene and unorthodox approach to treatment like use of unconventional ear drops and concoctions such as oil and honey into the middle ear may initiate the proliferation of opportunistic pathogens leading to blockage of Eustachian tube [16].

In the present study Staphylococcus aureus was found to be the most predominant organism $54.54 \%$ followed by Proteus spp $13.61 \%$ and Pseudomonas aeruginosa $12.73 \%$. Higher prevalence of $S$. aureus in this study also resembled very much with Park et al having 54\% of $S$. aureus [17]. The result is also in harmony with other studies $[9,13,18-20]$. In contrast, Gul et al and Iqbal et al isolated higher proportion of $P$. aeruginosa $[21,22]$.The reason behind $S$. aureus to be most prevalent organism might be because it is an opportunistic pathogens and a normal flora of skin, but when it gains entrance into the human body it causes infection to tissues and mucous membrane [23]. In different previous studies [16, 24, 25], anaerobic bacteria were also isolated but we have not included anaerobic bacteria in this study.

Thirty one $(21.98 \%)$ fungal isolates were obtained in the present study which was similar to the previous study carried out by $\mathrm{Nia}$ et al in which $24.57 \%$ of fungi were isolated [20].It has been postulated that the prolonged use of topical broad spectrum antibiotics leads to suppression of bacterial flora and subsequent emergence of opportunistic fungal flora in the areas of oral cavity, gastrointestinal tract and vaginal tract. Likewise in case of the middle ear, fungal infection supervenes because of prolonged use of topical antibiotics. It happens because of settling of fungal elements like spores from external environment in the moist and alkaline medium of middle ear discharge and debris. This finally leads to the development of mycotic otitis media causing intractable otorrhoea [26]. Of the fungal isolates, Aspergillus spp. predominated which was similar with the study conducted by Shrestha et al and Loy et al but in contrast, Parveen and Rao found Candida spp. to be the most common fungal isolates whereas only Candida spp. were isolated in the study done by Nwabuisi and Ologe $[11,13,20,27]$.

In this study almost all bacterial isolates were found to be sensitive toward gentamycin. Study done in Bir hospital and Om hospital, Nepal also found gentamycin as one of the effective antibiotic $[14,28]$. In the present study $S$. aureus the most predominated organism had high sensitivity toward cloxacillin and gentamycin. The result is similar to the restrospective study carried out in Bharatpur, Nepal [29]. Of the $\mathcal{S}$. aureus isolated, $4(6.67 \%)$ were methicillin resistant which was similar with the result of Iqbal et al [22]. Isolation of MRSA could be community acquired infection as all the patients included were out patients [22].

\section{Conclusion}

Staphylococcus aureus was the most common isolates and was most sensitive to Cloxacillin followed by Gentamycin and Chloramphenicol. For overall bacterial isolates Gentamycin was found to be the most effective drug. Aspergillus $s p p$. was the mostly isolated fungal isolates of which $A$. fumigatus predominated.

\section{Acknowledgement}

We are grateful to all staffs of Dhulikhel Hospital for their kind co-operation throughout this work. And we are also thankful to University Grant Commission for providing grant. 


\section{REFERENCES}

1. World Health Organization. Chronic suppurative otitis media: Burden of illness and management options [online] Geneva: World Health Organization. Child and Adolescent Health and Development Prevention of Blindness and Deafness 2004.

2. PooreyV.K and Iyer A. Study of bacterial flora in CSOM and its clinical significance. Indian Journal Otolaryngol Head Neck Surg 2002; 54 (2):91-5.

3. Shrestha R, Baral K, Neil W. Community ear care delivery by community ear assistants and volunteers: a pilot study. J LaryngolOtol 2001; 115:869-73.

4. Alsaimary IE, Alabbasi AM and Najim JM. Impact of multi drugs resistant bacteria on the pathogenesis of chronic suppurative otitis media. Afr J Microbiol Res 2010; 4 (13):1373-82.

5. Srivastava A, Singh RK, Varshney $S$, Gupta P, Bist SS, Bhagat S and Gupta N. Microbiological evaluation of an active tubotympanic type of chronic suppurative otitis media. Nepalese Journal of ENT Head and Neck Surgery 2010; 1 (2): 14-6.

6. Sharma K, Aggarwal A and Khurana P.M.S. Comparison of bacteriology in bilaterally discharging ears in chronic suppurative otitis media. Indian J Otolaryngol Head Neck Surg 2010;62 (2): 153-7.

7. CLSI. Performance standard for antimicrobial susceptibility testing; Twenty-First Informational Supplement. CSLI document M100-S12, Wayne, PA. Clinical and Laboratory Standard Institute (2011).

8. Kumar $\mathrm{H}$ and Seth S. Bacterial and fungal study of 100 cases of chronic suppurative otitis media. J Clin Diagn Res 2011; 5 (6): 1224-7.

9. Lodhi M, Munir T, Aziz K and Lodhi H. Chronic suppurative otitis media; Empiric quinolones in children. Professional Med J 2010; 17 (3):420-4

10. Yousuf M, Majumder K.A, Kamal A, Shumon A.M and Zaman Y. Clinical study on chronic suppurative otitis media with cholesteatoma. Bangladesh J Otorhinolaryngol 2011; 17 (1):42-7.

11. Loy AHC, Tan AL and Lu PKS. Microbiology of chronic suppurative otitis media in Singapore. Singapore Med J 2002; 43 (6): 296-9.

12. Mansoor T, Musani MA, Khalid G and Kamal M. Pseudomonas aeruginosa in chronic suppurative otitis media: Sensitivity spectrum against various antibiotics in Karachi. J Ayub Med Coll Abbottabad 2009; 21(2):120-3.

13. Shrestha BL, Amatya RCM, Shrestha I and Ghosh I. Microbiological profile of chronic suppurative otitis media. Nepalese J ENT Head Neck Surg 2011; 2 (2):6-7.

14. Jha AK, Singh JB and Dutta D.Microorganisms present in discharging otitis media in a group of patients in Kathmandu. Nepal Med Coll J 2007; 9 (3).

15. Nwabuisi C and Ologe FE. Pathogenic Agents of Chronic Suppurative Otitis Media in Ilorin, Nigeria. East Afr Med J 2002; 79 (4):202-5.

16. Prakash R, Juyal D, Negi V, Pal S, Adekhandi S, Sharma $M$ and Sharma N. Microbiology of Chronic Suppurative Otitis Media in a Tertiary Care Setup of Uttarkhand State, India.N Am J Med Sci 2013;5 (4).

17. Park DC, Lee SK, Cha CI, Lee SO, Lee MS and Yeo SG. Antimicrobial resistance of Staphylococcus from otorrhea in chronic suppurative otitis media and comparison with results of all isolated Staphylococci. Eur J Clin Microbiol Infect Dis 2008; 27 (7):571-7.

18. Haider A. Chronic suppurative otitis media (CSOM): Bacteriological study. Orion Med J 2002; 13: 13-4.

19. Ayson P.N, Lopez J.E.G and Lianes E.G.DV. Chronic Suppurative Otitis Media: Bacteriology and Drug Sensitivity Patterns at the Quirino Memorial Medical Center (20042005): A Preliminary Study. Philipp J Otolaryngol Head and Neck Surg 2006; 21(1, 2):20-3.
20. Nia K.M, Sepehri G, Khatmi H and Shakibaie MR. Isolation and Antimicrobial Susceptibility of Bacteria from Chronic Suppurative Otitis Media Patients in Kerman, Iran. Iranian Red Cresecent Med J 2011; 13 (12): 891-4.

21. Gul A.A, Rahim E, Ali L and Ahmed S. Chronic suppurative otitis media; frequency of Pseudomonas aeruginosa in patients and its sensitivity to various antibiotics. Professional Med J 2007; 14 (3):411-5.

22. Iqbal $K$, Khan $M$ and Satti L. Microbiology of Chronic suppurative otitis media: Experience at Dera Ismail Khan. GJMS 2011; 9 (2): 189-93.

23. Alo M.N, Anyim C, Okonkwo E.C and Orji J.O. Prevalence, Antibiogram of Bacterial Pathogens Associated with Otitis Media among Primary School Children in Ebonyi State. J Pharm Bio Sci 2012; 1 (78):17-20.

24. Brook I and Santosa G. Microbiology of chronic suppurative otitis media in children in Surabaya, Indonesia. Int J Pediatr Otorhinolaryngol 1995; 31: 23-28.

25. Ibekwe A.O, Shareef Z.A and Benayam A. Anaerobes and fungi in chronic suppurative otitis media. Ann OtolRhinolLaryngol 1997; 106(8): 649-652.

26. Mittal A, Mann S.B.S, Panda N.K, Mehra Y.N and Talwar P. Secondary fungal infections in chronic suppurative otitis media. IJO \& HNS 1997; 49(2): 112-6.

27. Parveen S.S and Rao J.R. Aerobic bacteriology of Chronic Suppurative Otitis Media (CSOM) in a teaching hospital. J Microbiol Biotech Res 2012; 2 (4): 586-9.

28. Aryal C, Adhikari S and Shrestha J. Bacteriological study of ear discharge in Bir Hospital. J Nepal Med Assoc 2002; 41:318-22.

29. Sanjana R.K, Singh Y.L and Reddy N.S. Aerobic bacteriology of Chronicsuppurative otitis media $(\mathrm{CSOM})$ in a tertiary care hospital: A restrospective study. Nepal Med Coll J 2011;7(2):1-8. 